\title{
Simulation of the Acceleration of Polarized Protons In Circular Accelerators
}

\author{
Yu.Shatunov, V. Yakimenko, \\ Budker Institute of Nuclear Physics, Novosibirsk, 630090, Russia
}

\begin{abstract}
An acceleration of high energy polarized protons is a long term issue of spin phisics. The application of Lie algebra method for the calculation of the strength spin resonanses (both intrinsic and imperfection) in the high energy proton ring is discussed. The simulation results of the polarized proton acceleration in RHIC with two siberian snakes are presented.
\end{abstract}

\section{Introduction}

Beam polarization can be lost during acceleration, when the particles pass the spin resonance. These resonances appear in the case, where the spin precession number $G \gamma(G=1.793$ is the anomalous magnetic moment for protons) is the integer or is equal to $k P \pm Q_{y}$. Here $P$ is the number of superperiods, $Q_{y}$ is the vertical betatron frequency, and $k$ is the integer. Depolarization (in the case of the verticaly polarized beam) is caused by horizontal magnetic fields being always present in the rings. Under resonance conditions, the action of these fields is coherent, and they force the spin to deviate from the stable vertical direction. The spin-betatron resonances are due to the horizontal fields generated by the vertical betatron motion.

During proton acceleration at RHIC[5] from a $20 \mathrm{GeV}$ injection energy to a $250 \mathrm{GeV}$ experimental energy, the beam passes several thousand spin resonances. The most important intrinsic resonances are approximately within $G \gamma=k P \pm Q_{y} \approx$ $m P M \pm Q_{b}$, where $k$ and $m$ are the integers, $P$ is the superperiodicity, $M$ is the number of the FODO cells in the superperiod, and $2 \pi Q_{b}=\left(2 \pi Q_{y}-6\right)$ is the phase advance in all the FODO cells containing the bending magnets. Position of the three most powerful resonances is the following:

\begin{tabular}{|c|c|}
\hline$G \gamma$ & $E[\Gamma \ni \mathrm{B}]$ \\
\hline \hline $3 \times 81+\left(Q_{y}-6\right)$ & 139 \\
$5 \times 81-\left(Q_{y}-6\right)$ & 200 \\
$5 \times 81+\left(Q_{y}-6\right)$ & 224 \\
\hline
\end{tabular},

where 81 is the product of the superperiodicity (3) and the effective number of the FODO cells in the superperiod (27), which comprise insertions for dispersion suppression. The simulation results are given for $E=224 \mathrm{GeV}$. The most important imperfection resonances are near the powerful intrinsic ones. Therefore, the analysis of the region $E=224 \pm 2 \mathrm{GeV}$ considers all the important aspects.

There are a lot of studies devoted to description of depolarization due to the isolated spin resonance crossing [1]. However, with a sufficiently large force the resonances become overlapping, so the analytical results do not work. There is a possibility of suppressing these resonances using the Siberian snakes [2]. However, the predicted so called "snake's resonances" [3], [4] expected at sufficiently powerful ordinary spin resonances require the special examination. The contradictory data obtained by a model tracking [3] impelled us to use the code SpinLie [6] to simulate the conservation of polarization during the proton beam acceleration at RHIC with the Siberian snakes.

\section{Simulation Model}

The simulation was linear in order to save the computer time. The betatron frequencies were $Q_{x}=28.19$ and $Q_{y}=29.18$, respectively. For resonance strength estimation we introduced next mashine imperfections: quads rotation angles relative to the longitudinal axis and quads vertical displacement have Gaussian distributions (truncated at two standard deviations) and with the amplitudes of $0.3 \mathrm{mrad}$ and $0.25 \mathrm{~mm}$, respectively. These imperfections cause the RMS orbit disturbance of about a $8 \mathrm{~mm}$. The residual after correction RMS orbit deviation was approximately a $0.3 \mathrm{~mm}$. The normalized emittance (according to [5]) was assumed to be equal to $\epsilon_{n x}=\epsilon_{n y} \equiv 6 \pi \beta \sigma_{x, y} / \beta_{x, y}=20 \pi$ mm mrad.

\section{Simulation Results}

The spin resonance force [1] can be characterized by the dependence of the root-mean-square spin spread $\Delta \vec{n}_{r m s} \equiv$ $\left\langle\left(\vec{n}(\theta, \vec{z})-\vec{n}_{0}(\theta)\right)^{2}\right\rangle^{1 / 2}$, where $\vec{n}_{0}(\theta)$ is the periodic spin solution for a synchronous particle on an ideal orbit and $\vec{n}(\theta, \vec{z})$ is the periodic spin solution for a off-exis particle, $<>$ denote the averaging over the beam distribution. In this notations a resonance strength is the half-width of the dependence $\Delta \vec{n}_{r m s}$ on the level $\Delta \vec{n}_{r m s} \approx 0.6$, which corresponds to the angle between the vectors $\vec{n}$ and $\vec{n}_{0}$ of the order $\pi / 4$. Figure 1 illustrates the simulation of the imperfection resonances (for $\epsilon_{n x}=\epsilon_{n y}=0$ ). The dashed curve in this figure shows complete suppression of these resonances by two Siberain snakes, which are placed diametrically opposite in the collider magnet system and rotate the spin by $\pi$ relative to the longitudinal and horizontal axes, respectively.

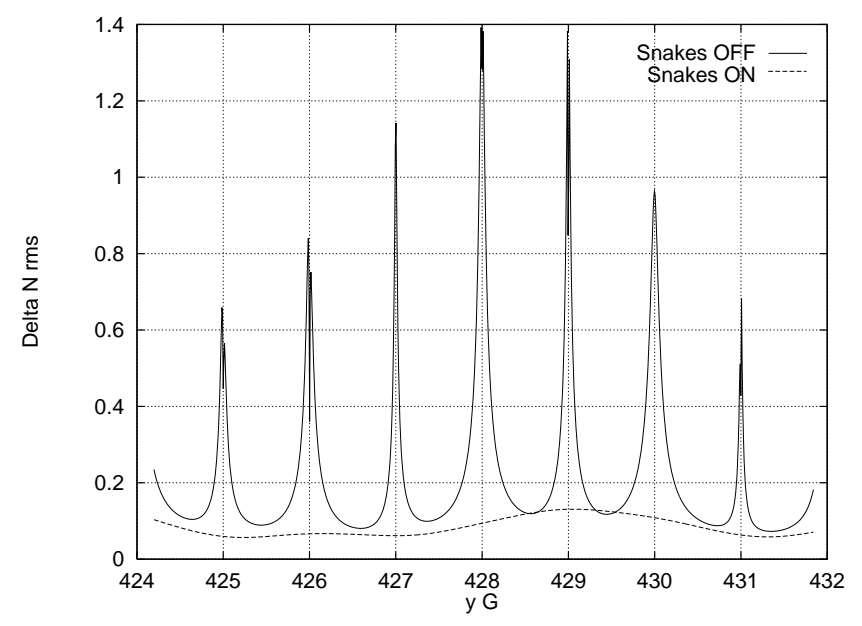

Figure. 1. Imperfection resonances and their suppression by two Siberian snakes. 


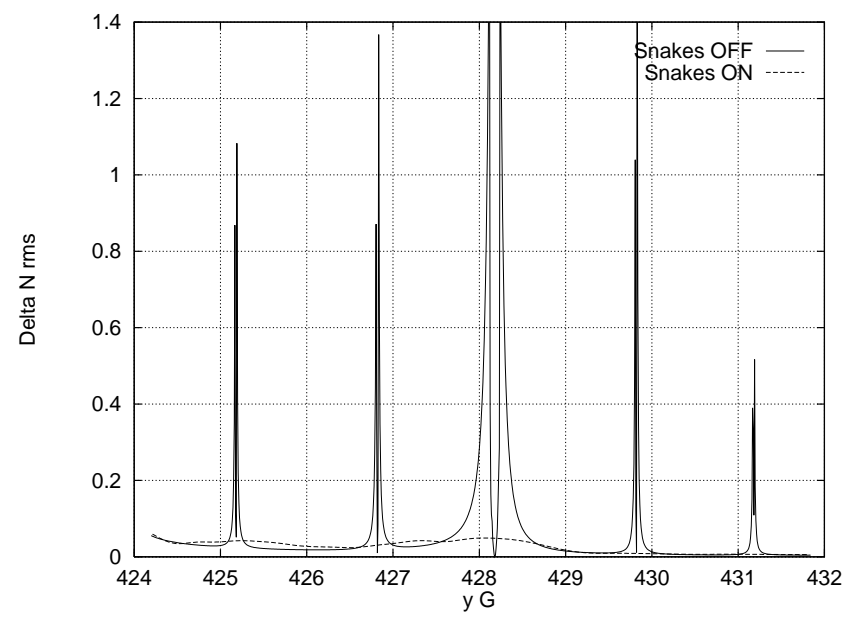

Figure. 2. Intrinsic resonances without orbit disturbances and their suppression by two Siberian snakes

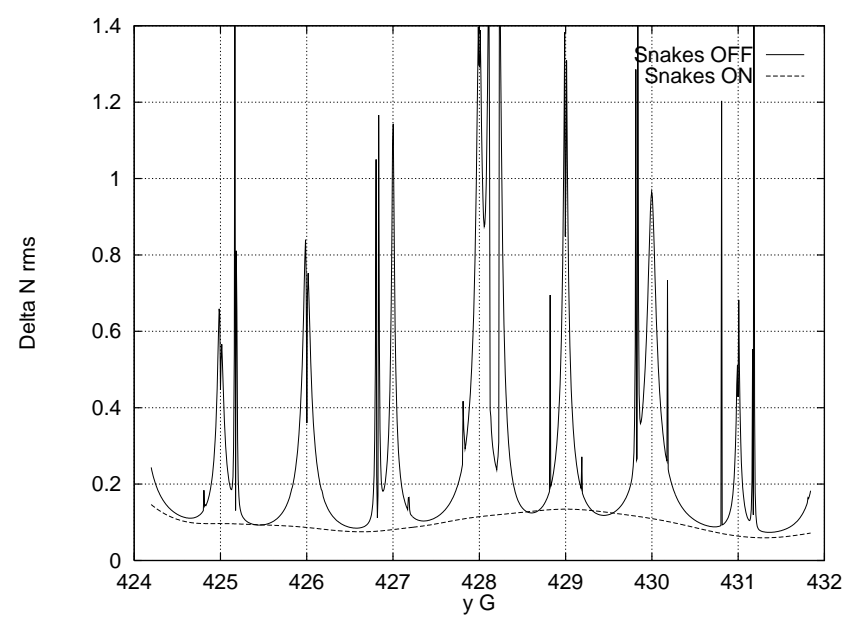

Figure. 3. Intrinsic and imperfection resonances, and their suppression by two Siberian snakes

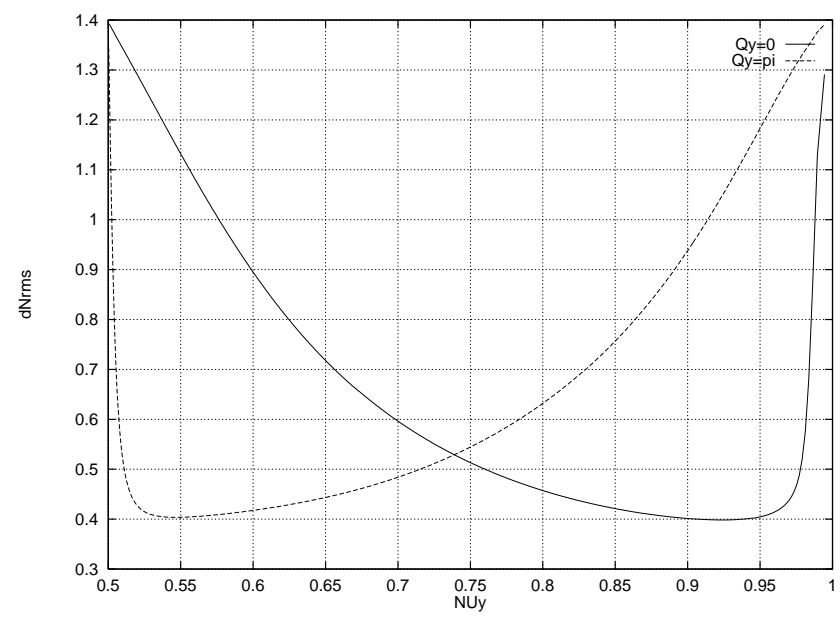

Figure. 4. The root-mean-square spin spread versus vertical betatron frequency under the resonance condition $Q_{\text {spin }}=399+$ $Q_{y}$. Snakes ON. Two cases are presented: the vertical phase advantage on snakes are 0 and $\pi$ correspondently.
Figure 2 shows the simulation of the intrinsic resonances without C.O. disturbances with two Siberian snakes on and off (for $\left.\epsilon_{n x}=\epsilon_{n y}=20 \pi\right)$. The joint of the intrinsic and imperfection resonances with two Siberian snakes on and off is presented in Fig. 3.

In calculations, the snake is assumed to be ideal, i.e., it is a thin snake with a zero betatron phase advance $\left(\Delta Q_{x}=0\right.$ and $\Delta Q_{y}=0$ ). The length and phase advance on the snake should be specially studied. As Fig. 4 shows, the different phase advance is favorable for different values of the full betatron frequency. This figure presents the root-mean-square spin spread versus the vertical betatron frequency under the resonance condition $Q_{s p i n}=399+Q_{y}$ (expected spin resonance point). Two curves correspond to two values of the vertical betatron phase advance on the snake $\Delta Q_{y}=0$ and $\Delta Q_{y}=1 / 2$.

\section{Spin Tracking}

Additionally to estimation of the resonance strength Lie algebra technics was applied for spin tracking while particle accelerating. The below results are obtained in the assumption that the beam consists of 50 particles with the Gaussian distribution in the phase space. The particle coordinates are prescribed relative to a zero (ideal) orbit.

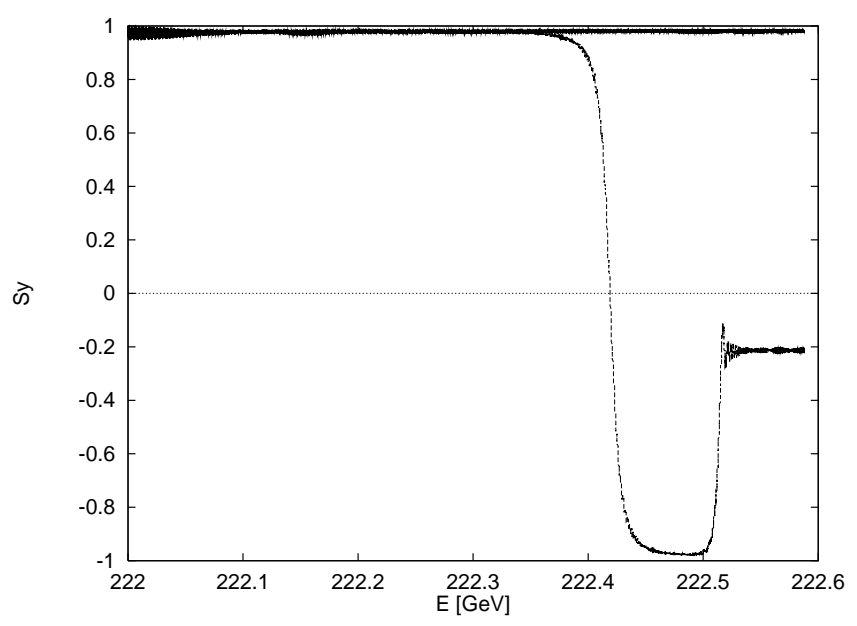

Figure. 5. Spin-flip due to crossing imperfection resonance $(\gamma G=425)$ and its canceling by two snakes

The acceleration rate of about $120 \mathrm{keV} / \mathrm{turn}$ is close to a lower boundary of the designed rate. The alinement errors and the orbit compensation are taken into account. Figure 5 shows a spin-flip due to the adiabatical imperfection resonance crossing and then strong depolarization from nearest intrinsic resonance. When Siberian snakes are on we did not see spin-flip and depolarization (upper curve). Corresponding beam spin spread is presened on Figure 6.

\section{Conclusion}

The obtained results show that the Lie algebra method is applicable to calculating the dynamics of a spin motion. Moreover, they confirm the abilitiy of the Siberian snakes concept and the possibility of a polarized proton beam acceleration at RHIC up 


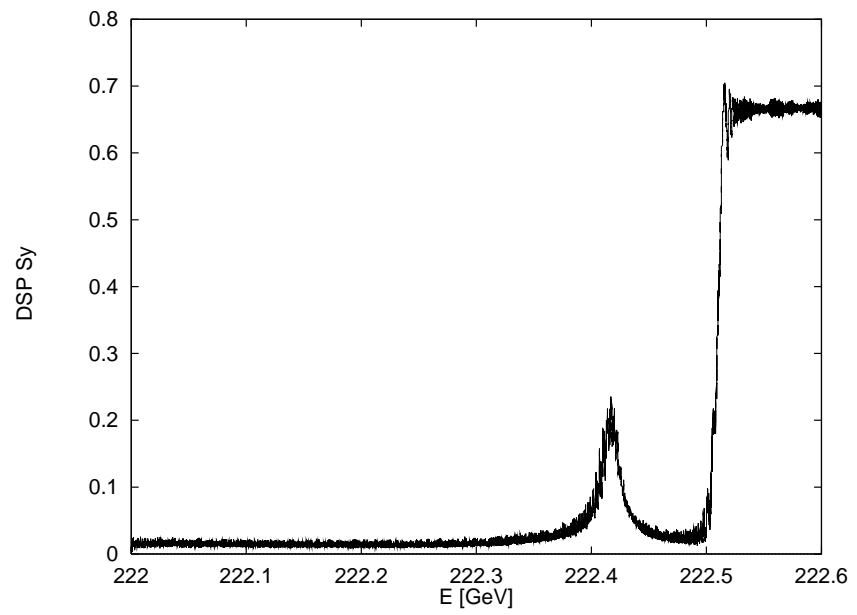

Figure. 6. Spin dispertion in the beam crossing imperfection resonance $(\gamma G=425)$

to top energy. However, the validity of the simulation depends on whether the conditions correspond to the real ones.

\section{References}

[1] E.D.Courant, R.D.Ruth Acceleration of Polarized Protons In Circular Accelerators, BNL Report 51270, UC-28 (1980).

[2] Ya.S.Derbenev and A.M.Kondratenko, JETP 35, 230 (1970).

[3] S.Y.Lee, S.Tepikian, Phys.Rev.Lett.,56,1635, (1986).

[4] S.Y.Lee, High Energy Spin Physics, AIP Conf Proc., No.187, p.1105, (1988).

[5] RHIC Design Manual, Brookhaven National Laboratory, Upton, (1994).

[6] Yu.Eidelman, V.Yakimenko, SPINLIE - New Code for Polarization Calculation, PAC93, Washington, 1994, pp.430433. 